\title{
Alzheimer's Detection through 3D Convolutional Neural Networks
}

\author{
Ryan Hogan, Christoforos Christoforou \\ Division of Computer Science, Mathematics and Science \\ St. John's University, Queens, NY 11432 \\ ryan.hogan16@stjohns.edu, christoc@stjohns.edu
}

\begin{abstract}
To inform a proper diagnosis and understanding of Alzheimer's Disease (AD), deep learning has emerged as an alternate approach for detecting physical brain changes within magnetic resonance imaging (MRI). The advancement of deep learning within biomedical imaging, particularly in MRI scans, has proven to be an efficient resource for abnormality detection while utilizing convolutional neural networks $(\mathrm{CNN})$ to perform feature mapping within multilayer perceptrons. In this study, we aim to test the feasibility of using three-dimensional convolutional neural networks to identify neurophysiological degeneration in the entire-brain scans that differentiate between $\mathrm{AD}$ patients and controls. In particular, we propose and train a 3D-CNN model to classify between MRI scans of cognitively-healthy individuals and $\mathrm{AD}$ patients. We validate our proposed model on a large dataset composed of more than seven hundred MRI scans (half AD). Our results show a validation accuracy of $79 \%$ which is at par with the current state-of-theart. The benefits of our proposed 3D network are that it can assist in the exploration and detection of $\mathrm{AD}$ by mapping the complex heterogeneity of the brain, particularly in the limbic system and temporal lobe. The goal of this research is to measure the efficacy and predictability of $3 \mathrm{D}$ convolutional networks in detecting the progression of neurodegeneration within MRI brain scans of $\mathrm{HC}$ and AD patients.
\end{abstract}

\section{Introduction}

Alzheimer's disease (AD) is a debilitating type of dementia that can affect a person's memory and cognitive behavior in both mild and extreme cases such as the inability to remember, focus, or even understand who you are (Yang et al., 2019). $\mathrm{AD}$ is a common disease that has no cure and worsens over time, typically occurring in individuals of 65 and older, making up 50-60\% of all dementia cases (Yang et al., 2019). The ability to cure AD is not currently known while treatments such as prescription medication and therapy have not shown to slow degeneration within the brain. Changes in the brain allow AD to damage neurons throughout multiple compartments dealing with memory and cognitive function, specifically in the limbic system, temporal lobe, hippocampus, and cerebral cortex. The damage of these neurons is not repairable and breaks the communication pathways that our brains use to create short-term memories and maintain continuous cognitive function. Although not being performed in this iteration of research, a common approach is the identification of mild cognitive impairment (MCI) in patients of early-stage AD (Pan et al., 2020). This is a critical stage of detection in which the disease can worsen and spread to other parts of the brain. Detection of AD in early stages (MCI) is an important task to develop treatment remedies and offer potential management to this overwhelming disease.

The most common method of tracking AD progression has been facilitated through magnetic resonance imaging (MRI) as a non-invasive tool for detecting changes in brain volumes throughout incremental scans. Machine Learning methods have been employed in the study of cognitive processes (Christoforou et al., 2010; Christoforou et al, 2013,; Christoforou et al., 2018) and have offered an alternate form of MRI scans analysis by utilizing algorithms such as Support Machine Vector (SVM) and Random Forest (RF) to perform classification tasks through linear SVM classification and majority voting in RF. Other approaches such as Region Of Interest (ROI) patch detection, biomarkers, and cerebral spinal fluid (CSF) are alternative methods for performing classification of $\mathrm{AD}$. However, there has been recent knowledge to suggest a potential lack of information in ROI patches as well as being error-prone and laborintensive (Lin et al., 2018). For this reason, ROI was not considered for this study despite being popular in some 3D models where whole-brain volumes are used, following a 3D patch for areas known to represent $\mathrm{AD}$ degeneration (Pan et al., 2020).

While under the same umbrella as machine learning, deep learning has initiated a more sophisticated approach by applying different methods such as convolutional neural networks (CNN), stacked auto-encoders (SAE), and deep 
belief networks (DBN) to extract low-level features within an image. For classification tasks, CNN's have proved to be a valuable application for extracting learnable parameters as well as feature mapping within medical imaging (Kumar et al., 2017). Moreover, the CNN explicitly takes an image as direct input to measure spatial information within adjacent pixels to assist in weightsharing and backwards propagation. For this model, the $\mathrm{CNN}$ will be used to extract voxel information contained in MRI scans without the need for manual selection of image features typically done in ROI patches. However, to the best of our knowledge, most CNN approaches proposed in this domain, rely on 2D- convolutional layers that require MRI scans to be processed as 2D slices, thus ignoring the 3D spatial-structure of MRI brain scans.

Processing MRI images as a 3D volume, instead of 2D slices, could potentially provide additional information that differentiates between $\mathrm{AD}, \mathrm{MCI}$, and $\mathrm{HC}$ groups, but it also introduces additional computational complexity in the model. In this paper, we explore the feasibility of a processing MRI image as a $3 \mathrm{D}$ volume, a $\mathrm{CNN}$ that utilized 3D convolutional layers. We apply our model to detect degeneration between MRI scans of both $\mathrm{HC}$ and $\mathrm{AD}$ patients to yield a binary classification between two classes.

\begin{tabular}{|c|c|c|}
\hline & AD & HC \\
\hline N & 375 & 375 \\
\hline Gender M:F & $162: 213$ & $225: 150$ \\
\hline Age (Mean:Stdv) & $\mathbf{7 4 . 6}: 7.75$ & $\mathbf{7 6 . 5}: 5.39$ \\
\hline
\end{tabular}

Table 1: Alzheimer's disease (AD) and healthy control patients (HC)

\section{Materials and Methods}

To evaluate the feasibility of our model, magnetic resonance imaging (MRI) scans were downloaded from the Alzheimer's Disease Neuroimaging Initiative. Founded in 2003 by lead investor Dr. Michael W. Weiner, ADNI has become a leader in the field of neuroscience research, with a focus on evaluating the progression of mild cognitive impairment (MCI) and Alzheimer's disease (AD) through MRI and positron emission tomography (PET) scans, biomarkers, and neuropsychological evaluations.

\section{Dataset}

The ADNI 1.5T collection was downloaded because of its preprocessing pipeline including 3D gradwarp and $\mathrm{B} 1$ nonuniformity correction to prepare the scans for further processing. Consisting of three-axis slices (axial, coronal, sagittal), a 3D image is created by combining all planes. A total of 750 three-dimensional scans were used and divided evenly between Alzheimer's Disease patients and a cognitively normal control group, respectively $(n=750$ scans: $\mathrm{AD}=375, \mathrm{HC}=375$ ). Dataset statistics are shown in Table 1.

The average age of participants was $75 \mathrm{y}$, comprising of $\mathrm{T} 1$ scans in the neuroimaging informatics technology initiative (NIFTI, nii) format. T1 scans were used to brighten tissues with a high-fat content (white matter), allowing them to be more visible in the scan, while watery gray matter structures tend to appear darker (Pan, et al, 2018). The detection of degenerative brain tissue throughout multiple regions of interest (ROI) such as the hippocampus and cerebral cortex offers a prospective indication of AD. This is done by utilizing volumetric data to obtain full brain mapping within an MRI. This collection of data from ADNI is commonly used in AD research and can be seen in (Christian et al., 2015) to facilitate training.

\section{MRI Preprocessing}

Preprocessing of the MRI data was carried out using the CAT12 Toolkit pipeline with default settings. The pipeline includes spatial normalization, affine regularization, MNI space registration, image smoothing, white matter (WM) segmentation, and skull extraction on all images. Cat12 maps the brain to yield a $3 \mathrm{D}$ voxel spaces representing a specific volume within the image of $(\mathrm{X}, \mathrm{Y}, \mathrm{Z})$ dimensions according to height, width, and depth. This creates volumetric three-dimensional data in the same process as (Pan, D et al., 2020). After processing, each image had a new dimension of $121 \times 145 \times 121$ voxels, with a spatial resolution of $1.5 \times 1.5 \times 1.5 \mathrm{~mm}^{3}$ for each voxel. Data normalization was performed through voxel intensity normalization which divides the original voxel value by the original maximum value to derive a new value between 0 and 1. A sample pre-processed image is shown in figure 1.

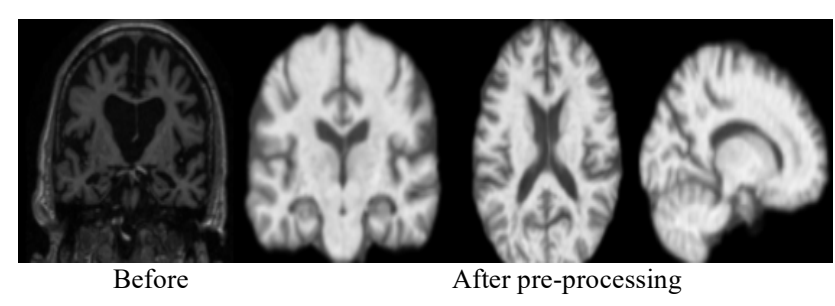

Figure 1: MRI image before (left) and after Cat12 WM segmentation, skull stripping and smoothing pre-processing with dimensions $121 \mathrm{x}$ $145 \times 121$ (shown in coronal, axial and sagittal view )

Image segmentation was then performed through White Matter (WM) tissue probability maps to identify white matter in the brain. White matter houses nerve fibers, known as axons, which carry electrical signals to other neurons and can show degeneration linked to AD. The pipeline used in this research follows the same procedure as (Pan, D et al., 2020) for 3D brain segmentation through 
the Cat 12 toolkit. Lastly, data augmentation was performed to reduce the opportunity of overfitting in the model by flipping images to promote disparity within the training set. Data augmentation is a necessary step in mitigating overfitting, particularly when having large imbalances between datasets. Several other steps can be taken to add augmentation such as translation, random noise, and scaling.

\section{Convolutional Neural Networks}

The CNN has displayed tremendous success as an image recognition tool that specializes in feature detection and can outperform its predecessors such as feedback neural networks by detecting learnable features within an image. In recent studies, 3D image classification has shown promising performance of $\mathrm{AD}$ classification through generic feature segmentation while using a $\mathrm{CNN}$ to reduce complexity (Yang, et al, 2019). The ability to detect features and inform a proper diagnosis has become integral in medical imaging to detect abnormalities within radiology scans such as MRI and PET (Gupta et al., 2019). Efficient diagnosis can offer alternative treatment plans such as medications and therapy that are typically accompanied by early detection of cancerous cells and degenerative tissues. By discretely taking images as input, the CNN will pass the image through multiple convolutional layers including filtering layers, pooling layers, and connected layers to provide a probabilistic output between 0 and 1 using the sigmoid function.

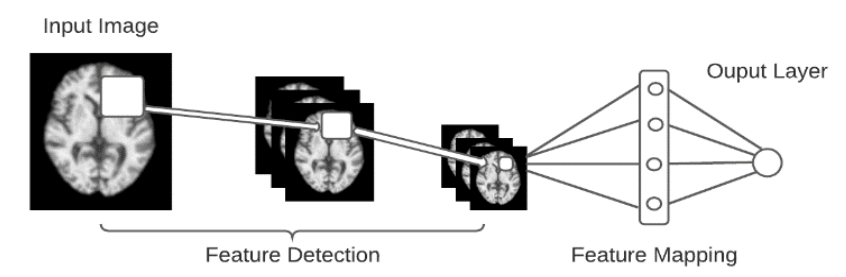

Figure 2: The above architecture shows how the CNN will take an image as input and map features throughout multiple convolutional.

CNN's have two main functions including feature detection and feature mapping to obtain learnable parameters by calculating adjacent spatial dimensions within an image (Yang, et al, 2019). Learnable filters (i.e., weights) are applied through feature extraction where each neuron is connected through receptive neurons of the previous layer to extract low-level features within an image (Yang, et al, 2019). Feature mapping is then performed by applying these filters as input to create a feature map. Down sampling along the spatial dimensions (X, Y, Z) allow for max-pooling and fully connected layers to be used, combining all neurons into a single dense output of
1. This process allows the $\mathrm{CNN}$ to effectively reduce the number of parameters in the network as well as the reduction of feature maps.

\section{Network Architecture}

Our proposed 3D-CNN architecture consists of four sequential layers of conv3D (with Relu activation function), max pooling, and batch normalization layers. Sequential convolutional filters of 32,64, 128, and 256 were used to extract learnable parameters within MRI scans to capture spatial and positional relationships throughout the brain. Each iteration of conv3D applies 3dimensional filtering (X, Y, Z) among the axial, coronal, and sagittal planes. Following is both max pooling, which down-samples the feature map to iteratively reduce the image size, and batch normalization to stabilize image weights and standardize inputs. The rectified linear unit (ReLU) activation function was used to apply a linear identity to our input by returning 0 representing a negative input and 1 as a positive output. Below is the network architecture:

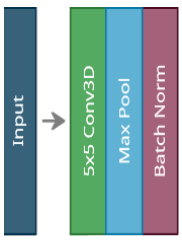

32 Filters

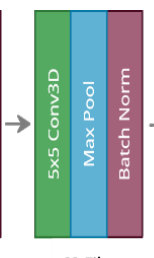

62 Filters

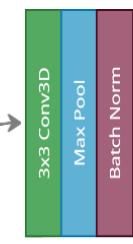

128 Filters

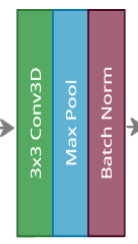

256 Filters

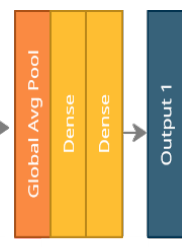

$512 \quad 1$
Figure 3: The CNN architecture used, consisting of four sequential layers of conv3D, max pool, and batch normalization.

Following the four sequential layers, global average pooling is used to calculate the average output for the feature maps of previous layers, concluding with a fully connected dense layer of 512 to flatten all neurons in the network. Lastly, a dense layer of 1 is used to combine all neurons into a final connected output of 1 . For the loss function, binary cross-entropy is used to yield a binary classification between two classes of HC and AD. Lastly, the sigmoid function is applied to find the probabilistic output of the model between 0 and 1 through binary classification. Dropout was used after the first and third convolutional layers as well as before and after the fully connected layers to reduce the risk of overfitting by randomly setting neurons in the previous layer to 0 after each iteration. Experiments were performed on a single node RTX 2060 Super GPU. Model performance was tested for 200 epochs with an average epoch computation time of 132 seconds, for a total runtime of eight hours.

\section{Classification Experiment}

A classification experiment between two classes, Alzheimer's disease (AD) patients and a healthy cognitive control group (HC) was performed to classify MRI scans 
as either $\mathrm{HC}$ or $\mathrm{AD}$ through binary classification. Consisting of 750 scans, an 80-20 train-test split was performed to utilize a training set for the model to learn features and a test dataset to perform validation against the train set. To avoid data leakage and promote image disparity, there was no overlap in scans between train and test classes. Binary classification was then performed between $\mathrm{HC}$ and $\mathrm{AD}$, yielding an accuracy of $79 \%$. Results of classification between $\mathrm{HC}$ and $\mathrm{AD}$ have shown to be at par or comparable to leading papers in this research such as (Pan et al. 2020), with an accuracy of $.84 \pm .03$ within a 2D CNN accompanied by ensemble learning (EL).

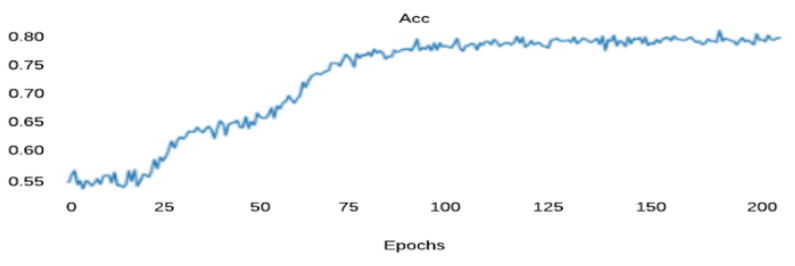

Figure 4: Model accuracy during training over 200 epochs.

\section{Performance Comparison}

The goal of this research was to show the efficacy of $3 \mathrm{D}$ convolutional networks in classification tasks while taking advantage of volumetric data to detect abnormalities. The results achieved offer insight into $3 \mathrm{D}$ models compared to other techniques such as support vector machine (SVM), random forest (RF), and principal component analysis (PCA) for classification tasks. The current results of our model show an accuracy that slightly outperforms the following study (Christian et al., 2015) when using SVM and PCA for classification tasks in MRI scans for AD detection ( $76 \%$ acc). To save time and computing costs, $2 \mathrm{D}$ models are also popular in classification tasks that take 2D images of either the axial, coronal, or sagittal planes to detect abnormalities within the angle of each plane. While being a simpler approach, 2D networks lack in extracting spatial information in non-volumetric data that is needed to efficiently map degeneration (Singh et al., 2020).

\section{Conclusion}

To inform a proper diagnosis and develop an understanding of Alzheimer's Disease progression, deep learning was used within a 3D convolutional architecture to provide an alternate approach for abnormality detection in MRI image data. The research provided offers attention to the efficacy and predictive power when detecting the progression of neurodegeneration within brain scans. The use of a volumetric data proves to yield classification accuracies comparable or better to other studies that ignore the third spatial dimension during training.
Alzheimer's disease continues to cause progressive issues within our society by overwhelming the physical and cognitive aspects of brain function that diminish a total sense of self. Although there is currently no cure, research in this field continues to grow and will hopefully facilitate future discoveries as well as the early detection of AD in MCI patients. Further research needs to be done to analyze the effectiveness of CNN's within medical imaging and neuroscience research, including the application between $3 \mathrm{D}$ architectures assisted by deep learning algorithms in its domain.

\section{References}

Christian, S.; Antonio, C.; Petronilla, B.; Gilardi, M. C.; Aldo, Q. and Isabella, C.; 2015. Magnetic resonance imaging biomarkers for the early diagnosis of Alzheimer's disease: a machine learning approach. Frontiers in neuroscience, 9, 307.

Christoforou, C.; Constantinidou, F.; Shoshilou, P.; and Simos, P. 2013. Single-trial linear correlation analysis: application to characterization of stimulus modality effects. Frontiers in Computational Neuroscience, 7, 15.

Christoforou, C.; Haralick, R.M.; Sajda, P.; and Parra, L. C. 2010. The bilinear brain: Towards subject-invariant analysis, In 2010 4th International Symposium on Communications, Control and Signal Processing (ISCCSP), pp. 1-6. IEEE, 2010.

Christoforou, C.; Hatzipanayioti, A.; and Avraamides, M. 2018. Perspective-taking vs mental rotation: CSP-based single-trial analysis for cognitive process disambiguation. In Wang, S., Yamamoto, V., Jianzhong S., Yang Y., Jones, E., Iasemidis, L., Mitchell, T., (Eds.) Proceedings of International Conference, Brain Informatics (pp. 109-199). Arlington, TX, USA.

Gupta, H.; Jin, K. H.; Nguyen, H. Q.; McCann, M. T.; and Unser, M. CNN-Based Projected Gradient Descent for Consistent CT Image Reconstruction, in IEEE Transactions on Medical Imaging, 37(6), 1440-1453.

Kumar, A.; Kim, J.; Lyndon, D.; Fulham, M., and Feng, D.; 2017. An Ensemble of Fine-Tuned Convolutional Neural Networks for Medical Image Classification in IEEE Journal of Biomedical and Health Informatics, 21(1), 31-40.

Lin, W.; Tong, T.; Gao, Q.; Guo, D.; Du, X.; and Yang, Y.; 2018. Alzheimer's Disease Neuroimaging Initiative. Convolutional neural networks-based MRI image analysis for the Alzheimer's disease prediction from mild cognitive impairment. Frontiers in neuroscience, 12, 777.

Pan, D.; Zeng, A.; Jia, L.; Huang, Y.; Frizzell, T.; and Song, X.; 2020. Early Detection of Alzheimer's Disease Using Magnetic Resonance Imaging: A Novel Approach Combining Convolutional Neural Networks and Ensemble Learning. Frontiers in neuroscience, 14.

Singh, S. P.; Wang, L.; Gupta, S.; Goli, H.; Padmanabhan, P.; and Gulyás, B.; 2020. 3D Deep Learning on Medical Images: A Review. Sensors, 20(18), 5097.

Yang, H.; Xu, H.; Li, Q.; Jin, Y.; Jiang, W.; Wang, J.; and Wang, T.; 2019. Study of brain morphology change in Alzheimer's disease and amnestic mild cognitive impairment compared with normal controls. in General Psychiatry, 32 